\title{
MÉTODOS DE PESQUISA EM ANÁLISE DO COMPORTAMENTO
}

\author{
Maria Amalia Pie Abib Andery ${ }^{1}$
}

Resumo: Apresenta-se neste artigo a discussão sobre o status da análise do comportamento como disciplina científica, bem como sua relação com outras ciências e as implicações desta discussão para a caracterização dos métodos de pesquisa em análise do comportamento. Várias classificações dos métodos de pesquisa são apresentadas, com destaque para o método experimental, tendo em vista seu papel na construção da disciplina. Os supostos e as principais características dos delineamentos de sujeito único são apresentados. E, por fim, são brevemente discutidas algumas questões que se originam da interação e aparente confronto entre conceituação e prática de pesquisa em análise do comportamento, tais como: reversibilidade comportamental, variabilidade comportamental, procedimentos de tentativa e procedimentos de operante livre, análise molecular e análise molar, tratamento estatístico e quantificação, pesquisa experimental com humanos.

Palavras-chave: Análise do comportamento. Método. Pesquisa experimental. Delineamentos de sujeito único.

\section{Métodos de Pesquisa em Análise do Comportamento}

É importantíssimo o papel que Maria Amélia Matos tem na história de inserção da análise do comportamento no Brasil desde a sua origem. Além da qualidade e diversidade de sua produção intelectual na análise do comportamento, sua participação na formação de gerações de pesquisadores foi essencial para a análise do comportamento no Brasil.Maria Amélia Matos marcou com sua prática como pesquisadora, professora e orientadora as maneiras de fazer análise do comportamento no Brasil (Matos,

1 A autora é bolsista de produtividade em pesquisa pelo CNPQ 
1996b, 1998). A pesquisa em análise do comportamento e a reflexão sobre a pesquisa no Brasil devem muito a Maria Amélia. Sua compreensão e compromisso com os supostos filosóficos da análise do comportamento e do behaviorismo radical, sua precisão no planejamento de pesquisa e sua perspicácia na análise de resultados deram qualidade ao seu trabalho e de seus orientandos e em boa medida estabeleceram parâmetros para a análise do comportamento no país. Neste artigo discute-se o escopo dos métodos de pesquisa em análise do comportamento e algumas de suas características mais marcantes. Assim como os padrões comportamentais individuais são sempre exemplos de leis do comportamento, assim também o comportamento de Maria Amélia e sua produção são exemplos da melhor prática de pesquisa e produção intelectual na análise do comportamento.

\section{Análise do comportamento como disciplina científica}

Em artigo publicado em 1980, Michael traça brevemente a história de desenvolvimento e crescimento da análise do comportamento, desde os trabalhos iniciais de Skinner nos anos 1930, destacando o percurso de institucionalização da análise do comportamento como campo do saber:o surgimento e a disseminação nos EUA de conteúdos e cursos de análise do comportamento, de material didático e de divulgação científica, congressos e periódicos cientíícos específicos do campo, e, finalmente, de novas práticas, em especial, o desenvolvimento de áreas de aplicação e da análise conceitual e de cunho filosófico associados à análise do comportamento (Michael, 1980). Michael revela claramente que a história do campo da análise do comportamento nos EUA em seus primeiros 50 anos é também uma história de ampliação e diversificação do campo, não apenas no sentido de crescimento ou ampliação dos conteúdos científicos, mas também de suas possibilidades de atuação e de suas maneiras de trabalhar.

Já seria tarefa complexa descrever e discutir o método de pesquisa que caracterizou os inícios da análise do comportamento, ou o trabalho de Skinner até o final dos anos 30 (Andery, Micheletto, \& Sério, 2002; Coleman,1985, 1987; Moxley, 2005; Smith, 1994). A diversificação e ampliação em termos de conteúdo e de possibilidades de produção de conhecimento que são característicos do campo tornam a tarefa ainda mais difícil. Para a discussão do(s) método(s) de pesquisa em análise do comportamento é necessário primeiro delimitar e caracterizar o que se chama hoje de análise do comportamento.

O primeiro ponto a destacar em tal delimitação diz respeito ao estatuto disciplinar da análise do comportamento. Há um debate não resolvido na análise do comportamento sobre o seu status e especificidade como ciência. Para alguns deveria ser assumida como disciplina independente, cujo objeto de estudo é o comportamento, e não como alternativa, perspectiva, ou corrente na psicologia, uma vez que a psicologia toma como 
objeto de estudo entidades distintas do fenômeno comportamental como, por exemplo, a psyché. ${ }^{2}$ Para outros a análise do comportamento é corrente/teoria/abordagem da psicologia, constituindo-se em alternativa a outras perspectivas exatamente porque, como as demais alternativas na psicologia, assumiu um objeto de estudo que é um fenômeno característico dos indivíduos; mas, diferentemente das demais abordagens, assumiu o comportamento em si mesmo como o fenômeno-alvo de seu interesse. Neste debate está em questão não apenas o caminho da institucionalização e profissionalização da análise do comportamento, mas também qual é o escopo dos problemas de pesquisa relevantes na análise do comportamento e qual seria o escopo de atuação dos profissionais que trabalham ou trabalharão com as técnicas e tecnologias desenvolvidas pela análise do comportamento. Temas relevantes e historicamente definidores da psicologia relacionados às práticas clínicas dos psicólogos, por exemplo, seriam considerados menos importantes para aqueles que defendem uma ciência independente e paralela à psicologia, mas não necessariamente para aqueles que tomam a análise do comportamento como alternativa conceitual no próprio campo da psicologia. Qualquer que seja a posição tomada diante deste debate, ele é revelador da complexidade do fenômeno que é nosso objeto de estudo e das possibilidades e dificuldades metodológicas enfrentadas na análise do comportamento hoje ${ }^{3}$ (Fraley \& Vargas, 1986, Keller \& Schoenfeld, 1950, Tourinho, 2003, 2006; Tourinho, Carvalho Neto, \& Neno, 2004; Vargas, 1987, 1989).

\section{A filiação da análise do comportamento em relação às ciências}

Outro debate relacionado, o qual parece estar cada vez mais a caminho de um acordo, diz respeito à caracterização da análise do comporta-

2 Skinner parece ter tido posição ambivalente sobre o tema. Assim, por exemplo, em Comportamento dos organismos (1938) propôs uma nova ciência: a ciência do comportamento, apresentando o livro como um sistema explicativo desta ciência, o que indicaria a proposição de uma ciência distinta das existentes até então, inclusive a psicologia. Outro possível indicador de que Skinner então supunha que a ciência do comportamento estaria vinculada à biologia e não à psicologia é sua discussão das relações entre esta ciência e a fisiologia. Entretanto, e assume-se aqui, não por acaso, Skinner estabelece no mesmo texto comparações entre sua proposta e a de autores como Tolman e Hull, que à época desenvolviam sistemas explicativos considerados como correntes alternativas à psicologia tradicional.

3 Keller e Schoenfeld publicaram, em 1950, o que se considera como primeiro texto didático de análise do comportamento. De fato, o sumário do livro e seu conteúdo oferecem uma sistematização dos princípios e leis que regulam o comportamento segundo a perspectiva da análise do comportamento. No presente contexto destaca-se o título do livro, Princípios de psicologia, e o primeiro objetivo proposto pelos autores: "familiarizar [o leitor] com um conjunto de princípios psicológicos bem-estabelecidos"(p. 1). 
mento como ciência natural ou como ciência do âmbito das ciências biológicas. No primeiro caso, destaca-se a filiação metodológica da análise do comportamento aos modelos de pesquisa consagrados nas ciências naturais e, mais especificamente, à pesquisa experimental de laboratório. No segundo caso, destaca-se que o objeto de estudo da análise do comportamento é ele mesmo um fenômeno dos seres vivos e destaca-se também o modelo de causalidade de variação e seleção assumido por Skinner (1981), que é também o modelo hegemônico na biologia hoje (Andery, 1997; Blackman, 1991; Johnston \& Pennypacker 1993a; Matos, 1999c, 2000; Micheletto, 2000; Moxley, 1989, 2001, 2002, 2003, 2005, 2007; Smith, 1994).

O debate é relevante porque a caracterização da análise do comportamento como ciência natural em oposição às chamadas ciências sociais centra-se em uma discussão metodológica que opõe métodos de pesquisa experimental a métodos de pesquisa chamados de interpretativos (ver J Johnston \& Pennypacker, 1993b). Esta discussão é também relevante porque a caracterização da análise do comportamento como ciência natural, que se baseia especialmente na obra mais inicial de Skinner (Skinner, 1938, 1947, 1953), implicou em interpretações - não necessariamente "autorizadas" pelo trabalho de Skinner - que valorizavam ou até mesmo chancelavam apenas a pesquisa experimental como modelo de pesquisa aceitável e que acabaram por possibilitar ou por servir de fundamento para interpretações errôneas de que a análise do comportamento seria uma ciência comprometida com posições antiteóricas, ou com posições positivistas, neopositivistas ou operacionistas (Andery \& Sério 1987, 1989; Micheletto \& Sério, 2002; Matos, 1999a, 1999c).

Já a defesa da ciência do comportamento como parte do campo das ciências biológicas centra-se na interpretação de que as ciências se "assemelham","inter-relacionam", ou se "aparentam" em função de pressupostos mais gerais: no caso presente, pela definição de seus objetos de estudo e modelos causais. A filiação da análise do comportamento à biologia darse-ia sobre bases que poderiam ser consideradas mais restritivas, porque comprometem a análise do comportamento com um modelo de causalidade que é aplicável aos seres vivos. Por outro lado, tal filiação, e especialmente o desenvolvimento da biologia em uma perspectiva selecionista, ampliaria as alternativas metodológicas chanceladas como produtivas e necessárias ao desenvolvimento da análise do comportamento para além da pesquisa experimental de laboratório. Nestes termos, argumenta-se que assim como as várias disciplinas da biologia beneficiaram-se de métodos de pesquisa não experimentais, como ocorreu, por exemplo, na genética de populações, a análise do comportamento também pode se beneficiar de métodos e procedimentos de pesquisa alternativos (Andery, 1997; Andery \& Sério, 1989; Catania, 1984; Glenn, 2003; Hull, Langman, \& Glenn, 2001; Moxley, 2001; Morris, Todd, Midgley, Schneider, \& Johnson, 1990). 
Embora não se possa afirmar que esta segunda perspectiva seja unanimidade entre aqueles que discutem a filiação científica e filosófica da análise do comportamento (ver Todd \& Morris, 1995), o seu próprio desenvolvimento como ciência pode ser tomado como indicativo de que a análise do comportamento de fato situa-se no campo das ciências biológicas.

\section{O escopo da análise do comportamento}

Estes debates e suas implicações para a discussão dos métodos de pesquisa em análise do comportamento estão em parte relacionados com o desenvolvimento das expressões que historicamente designaram a ciência que se desenvolveu a partir do trabalho de Skinner. Nos anos 1930 e mesmo em décadas subsequentes Skinner utilizava com frequência a expressão "ciência do comportamento" (Skinner, 1931, 1935, 1938, 1953). Outras expressões, cunhadas por Skinner e por outros passaram a ser empregadas no decorrer dos anos, tais como behaviorismo, behaviorismo radical, análise experimental do comportamento e, mais recentemente, análise do comportamento aplicada. Mas, especialmente com o advento do periódico Journal of the Experimental Analysis of Behavior, cujo título tem como referência o subtítulo de The behavior os organisms, ganhou força a expressão análise experimental do comportamento. No entanto, as restrições impostas por tal rótulo acabaram por dar lugar à expressão "análise do comportamento", que é mais inclusiva que análise experimental do comportamento e ao mesmo tempo permite a distinção entre ciência e filosofia que Skinner defendeu quando distinguiu behaviorismo e ciência do comportamento (Skinner, 1974).

Nestes 80 anos de desenvolvimento da análise do comportamento 4 ampliaram-se e tornaram-se mais complexos seu sistema teórico, os problemas de pesquisa investigados, as possibilidades de aplicação, a comunidade de cientistas e praticantes e os marcos de sua institucionalização como disciplina. A ampliação de complexidade e de escopo da disciplina marca-se por um duplo movimento.

De um lado cresceram e diversificaram-se os interesses e compromissos dos cientistas e praticantes, as possibilidades de interpretação de fenômenos e processos e os modelos de investigação utilizados. Um exemplo destes movimentos são as várias interpretações oferecidas na história da análise do comportamento e hoje existentes para conceitos fundamentais que são as noções de formação de classes de estímulos e os processos relacionados de reforçamento e discriminação (de Rose, 1993; Goldiamond, 1975; Matos, 1981b, 1999b; Sidman,1990, 1997; Skinner,1935,

4 Tomando-se como marco que inaugura a análise do comportamento - arbitrário, em certo sentido - o ano de 1931 e a publicação do primeiro artigo de Skinner em que o autor faz uma proposta para uma ciência do comportamento (The concept of reflex in the description of behavior). 
1956, Schick, 1971; Zentall, \& Smeets, 1996). Outro exemplo é o debate também central e que se trava, desde os anos 50 e 60, sobre o status, enquanto processo comportamental, da punição e controle aversivo, bem como a discussão dos procedimentos de investigação e dos resultados da pesquisa da área (Catania, 1999; Matos, 1981a; Sidman, 1989; Skinner, 1953). Os dois exemplos são significativos porque envolvem questões atuais que emergem elas mesmas do desenvolvimento conceitual e dos resultados de pesquisa e dos métodos de pesquisa empregados sistematicamente na disciplina. Mostram também as possibilidades e a vitalidade da análise do comportamento. Interessantemente, mas não por coincidência, em sua história como pesquisadora, Maria Amélia Matos contribuiu de maneira significativa para ambos os debates. Como pesquisadora, investigou fenômenos como supressão condicionada quando seus interesses de pesquisa estavam focados na questão do controle aversivo e dos subprodutos da punição e reforçamento negativo (Matos,1969, 1981a). Trabalhou também na área de controle de estímulos e contribuiu para a discussão dos processos e procedimentos de estabelecimento de controle discriminativo do comportamento operante (Matos, 1981b, 1999b; Matos \& Malerbi, 2001) e a formação de classes de estímulos (Debert, Matos, \& Mcllvane, 2007; Matos \& Hubner, 1992; Matos \& Lopes Jr, 1999; Matos, Hubner, Serra, Basaglia, \& Avanzi, 2003). Em preciosos artigos sobre ambos os temas, Maria Amélia Matos expôs de maneira aparentemente simples, porém sistemática, crítica e provocante, questões centrais em ambas as áreas de investigação e implicações dos resultados acumulados de pesquisa em ambas as áreas (Matos, 1981a, 1981b, 2002).

Por outro lado, tomando-se como exemplo ainda estas áreas e o trabalho de Maria Amélia Matos, a expansão, diversificação e as várias alternativas interpretativas e metodológicas (nem sempre são complementares) que emergiram no desenvolvimento da análise do comportamento contribuíram para um tipo de "especiação". Aparentemente a própria variabilidade que emergiu na disciplina ao longo dos anos conduziu a um conjunto - explícito e implícito - de características ainda difusas e pouco definidas que genericamente servem de base para que reconheçamos um pesquisador, pesquisa, trabalho publicado, prática profissional ou periódico como de análise do comportamento. Não é por outra razão que em suas publicações de 1981 e 1992, Maria Amélia Matos enfatiza a especificidade do tratamento conceitual e metodológico da análise do comportamento em relação aos temas de controle aversivo (1981a), de controle de estímulos (1981b) e formação de classes de estímulos por equivalência (2002).

Seja tomada como ciência independente ou como abordagem da psicologia, nestes termos, a análise do comportamento constitui-se em disciplina científica que se caracteriza pela definição de seu objeto de estudo, pelas concepções de conhecimento, mundo e homem que informam suas práticas e pelos resultados - empíricos, teóricos e tecnológicos - de sua 
atuação. Caracteriza a análise do comportamento seu objeto de estudo, o comportamento, que é definido como relação indivíduo-ambiente (Skinner, 1938, 1953, 1974). Mas também são características as maneiras especiais de abordar o fenômeno, ou seja, seus métodos de pesquisa (Moore \& Cooper, 2003).

A expressão "análise do comportamento" designa, então, um conjunto de práticas de uma comunidade (os analistas do comportamento) e seus produtos. Tais práticas envolvem as maneiras de fazer pesquisa e os seus resultados, ou seja, envolvem a pesquisa científica que serve de base e fundamento para a produção de corpo de conhecimento teórico e de explicações (comportamento verbal) sobre o comportamento e, então, para o desenvolvimento de técnicas, procedimentos e tecnologias de intervenção que são aplicadas para a solução de problemas envolvendo comportamentos.

O conjunto de práticas que chamamos de análise do comportamento envolve, portanto, a análise experimental do comportamento - pesquisa básica e pesquisa aplicada -, a análise do comportamento aplicada e a filosofia que a elas se vincula - behaviorismo radical (Michael, 1980; Catania, 1984; Tourinho, 2003, 2006) ${ }^{.5}$

\section{O escopo da pesquisa e dos métodos de pesquisa na análise do comportamento}

A pesquisa em análise do comportamento não se restringe à pesquisa básica e os métodos de pesquisa característicos do fazer ciência na análise do comportamento não se restringem ao método experimental, ainda que a pesquisa experimental tenha especial relevância tanto com

5 Como ressaltou Michael (1980), o desenvolvimento de publicações arbitradas específicas da análise do comportamento é relevante indicador desta caracterização. O primeiro periódico de análise do comportamento, já um sinal de sua fertilidade e crescimento da disciplina e ao mesmo tempo de suas especificidades, foi o Journal of the Experimental Aanalysis of Behavior, que ainda hoje é dedicado especialmente (ainda que não exclusivamente) à pesquisa experimental e de processos. O JEAB foi iniciado em 1958. Dez anos depois se iniciou o Journal of Applied Behavior Analysis, dedicado principalmente à publicação de resultados de pesquisa - também experimental -, mas em contextos aplicados (de intervenção). Já na década de 1970 outros dois importantes periódicos foram inaugurados: um deles dedicado à discussão filosófica (Behaviorism) e outro dedicado à discussão de questões conceituais e históricas (The behavior Analyst). Desde o final dos anos 1960 e pela década de 1970 constata-se também a expansão da análise do comportamento e da pesquisa em análise do comportamento para além das fronteiras americanas, na inauguração de outras publicações arbitradas com outros focos específicos de áreas de atuação e pesquisa em que se insere a análise do comportamento nos EUA e em outros países. No Brasil, já neste período (mais uma vez com a participação ativa também de Maria Amélia Matos) inicia-se o ensino e a pesquisa em análise do comportamento e ocorre a publicação do primeiro periódico da disciplina, hoje extinto. 
relação à história da análise do comportamento até aqui como em relação às práticas de pesquisa atuais e ainda que a pesquisa de processos (que é característica da pesquisa básica) seja pedra angular e indissociável da disciplina.

Nos esforços de caracterização dos métodos de pesquisa considerados aceitáveis na análise do comportamento, especialmente na literatura americana, frequentemente as pesquisas são classificadas com base na origem de seu dados, como "data-based", que é a pesquisa que responde a um problema com produção de dados, os quais são coletados e analisados sistematicamente e segundo procedimentos bem-estabelecidos - ou não. Neste contexto excluem-se do rol de métodos de pesquisa valorizados aqueles que não são rotulados como "data-based". O problema é que o termo frequentemente é confundido com pesquisa básica experimental ou, em uma interpretação mais liberal, com a pesquisa em que a coleta de dados envolveu medidas comportamentais sistemáticas in loco. Em qualquer dos casos discute-se a validade de pesquisas de base observacional e corre-se o risco de classificar as pesquisas conceituais ou reflexivas da classificação como inaceitáveis.

Talvez uma expressão mais adequada para a distinção seria "pesquisa de base empírica", que se contraporia a pesquisa de base documental: inclui-se na pesquisa de base empírica a pesquisa observacional, enquanto que a pesquisa de base documental envolve a produção de dados que são produtos de comportamento verbal acumulado (na comunidade científica ou extra-científica). A pesquisa conceitual, a pesquisa histórica, enfim, a pesquisa reflexiva é sempre de base documental, ou seja, os dados que a suportam são os próprios resultados e o desenvolvimento científico de uma área, um tema, um conceito, um período etc. No entanto, mesmo esta segunda expressão é problemática. Afinal, a pesquisa teórica ou reflexiva também depende da coleta de dados (é, neste sentido, "data-based") e também tem, portanto, "base empírica". A confusão possivelmente se deve à tentativa de distinguir comportamento verbal, que é emitido pelo cientista com base em coleta e análise sistemática de dados (que ocorreria na pesquisa conceitual ou reflexiva),e de base documental, daquele comportamento verbal que é emitido independentemente de procedimentos sistemáticos e públicos de coleta e análise de dados e que muitas vezes é apresentado, incorretamente, como "pesquisa" conceitual (Micheletto, Andery, \& Sério, 2000; Morris, Todd, Midgley, Schneider, \& Johnson, 1990; Tourinho, 1999).

Além disso, o problema que muitas vezes não é explicitamente tratado quando se desconsidera ou critica a pesquisa de base documental não é se há ou não dados, mas que os dados envolvem comportamento verbal que é, ele mesmo, objeto de estudo de uma ciência do comportamento.Ou seja, o problema é que, na pesquisa reflexiva (como ocorre, aliás, em qualquer pesquisa que envolve questionamento - questionários e entrevistas) 
trabalha-se com um dado "secundário", com o relato do fenômeno - que é mais comportamento a ser explicado. Ou seja, o pesquisador tem problemas metodológicos, que são inerentes ao dado, quanto ao depósito seletivo de informações, e tem medidas indiretas do fenômeno de seu interesse. No entanto, tais problemas também podem ocorrer na pesquisa "de base empírica" e desde que sejam reconhecidos, considerados e metodologicamente tratados não invalidam a pesquisa conceitual ou histórica.

O exame de alguns anos consecutivos das pesquisas publicadas em periódicos classificados como de análise do comportamento, em livros e artigos de autores reconhecidos da disciplina (a começar por Skinner, 1950, 1957) e em publicações especificamente voltadas para questões filosóficas ou metodológicas (por exemplo, Moore, 2008; Matos, 1999a; Moxley, 1999; Todd \& Morris, 1995; Tourinho, 2003), indica que há variação nos procedimentos de coleta, mensuração do fenômeno observado e de análise e interpretação de dados utilizados na análise do comportamento. Esta variabilidade reflete uma das classificações mais usuais dos métodos de pesquisa. Encontra-se na análise do comportamento pesquisa: (1) experimental, envolvendo a manipulação de variáveis, e (2) "descritiva” ou "observacional", quando não há manipulação planejada de variáveis sob controle do pesquisador.

A pesquisa na análise do comportamento também pode ser classificada quanto às suas finalidades em termos da construção de conhecimento sobre o comportamento. Como tal, pode referir-se: (1) à descoberta ou descrição de processos comportamentais, caracterizando-se como pesquisa básica; (2) à descoberta ou descrição de conteúdos comportamentais que são selecionados em função de processos comportamentais, casos em que são pesquisas consideradas ora como básicas e ora como aplicadas; (3) à produção, descrição e/ou validação de procedimentos e técnicas que se fundamentam nas descrições de processos e conteúdos comportamentais originados de outras pesquisas, casos em que são rotuladas como pesquisa aplicada; (4) ou pode ter como objetivo a reflexão sobre a própria análise do comportamento e seu desenvolvimento, casos em que o dado é o próprio conhecimento produzido e a pesquisa é chamada de "conceitual" ou "histórica" ou "reflexiva." Portanto, a pesquisa em análise do comportamento pode ser voltada para a descrição de processos comportamentais, como ocorre mais frequentemente na pesquisa básica, pode ter como objetivo descrever e intervir em conteúdos comportamentais, ${ }^{6}$ o que em geral (mas não exclusivamente) é típico de pesquisa aplicada, e pode ser classificada como pesquisa reflexiva.

A pesquisa em análise do comportamento também pode ser classificada quanto ao setting em que é conduzida: pode ser baseada em laboratório, o que, em geral, é característico da pesquisa básica, ou pode ser reali-

6 Como alertou Luna (2007), entre outros, é importante evitar confusão entre pesquisa aplicada e prestação de serviços ou técnicas e procedimentos técnicos aplicados profissionalmente. 
zada como "pesquisa de campo", ou como pesquisa em "contextos naturais ou naturalísticos."Laboratório é apenas um setting em que são criadas condições, em geral simplificadas, para estudar o fenômeno de interesse, o que aumenta o controle sobre as variáveis de interesse do pesquisador. Embora o setting de laboratório seja mais tipicamente o setting da pesquisa de processos na análise do comportamento, há inúmeros exemplos na literatura em que pesquisa básica tem sido conduzida em settings naturais e em que a pesquisa aplicada tem sido conduzida em ambientes simplificados e protegidos mais típicos de situação de laboratório. O trabalho de Maria Amélia Matos e seus orientandos com controle de estímulos e alfabetização é exemplar destas situações: em um setting de laboratório coletou-se sistematicamente dados para responder a um problema de pesquisa que envolvia questões sobre discriminação e formação de classes de estímulos, mas com a preocupação de como tais processos participariam na produção de um dado repertório (conteúdo) comportamental, a aprendizagem de leitura e escrita (Matos \& Hubner, 1992; Matos, Hubner, Peres, \& Malheiros, 1997; Matos, Hubner, Serra, Basaglia, \& Avanzi, 2003).

Assim, hoje, a pesquisa em análise do comportamento tem distintas finalidades e modalidades, envolvendo diferentes métodos. E embora não se exija de um pesquisador particular proficiência ou prática de pesquisa em variadas modalidades, ou com os vários métodos de pesquisa, é indiscutível que nestes 80 anos de história da análise do comportamento pelo menos alguns dos mais proeminentes analistas do comportamento praticaram sua ciência de maneiras variadas no que se refere à consolidação e institucionalização do campo, mas também no que se refere à prática de pesquisa. O exemplo paradigmático é mais uma vez Skinner:ainda que seu nome seja mais frequentemente associado à pesquisa experimental e à pesquisa básica (de processos), Skinner foi um incansável pesquisador de temas que abordou em uma perspectiva reflexiva, ou conceitual (por exemplo, Skinner, 1945, 1957, 1974, 1981). Foi também pesquisador com interesses aplicados, especialmente nas áreas de educação e automanejo e planejamento cultural (Skinner, 1936, 1959a, 1959b, 1961, 1968, 1972, 1983). Finalmente, obras como Verbal behavior (Skinner, 1957), bem como artigos anteriores sobre o tema (Skinner, 1937, 1938b, 1939), mostram claramente o quanto Skinner se beneficiou de dados que envolveram a descrição de conteúdos sem qualquer manipulação.

No trabalho de Maria Amélia Matos encontram-se as mesmas características que são distintivas da formação da análise do comportamento como campo do saber e a mesma diversidade de interesses, de habilidades como pesquisadora e de recurso a métodos diversificados de pesquisa:em seu trabalho estão presentes a produção de conhecimento a partir de pesquisa básica (Matos, 1981b; Matos \& Lopes Júnior, 1999; Matos, Paracampo, Souza, \& Albuquerque, 2001) e também de pesquisa aplicada (Matos, 1993, 1999d Matos, Hubner, Serra, Basaglia \& Avanzi, 2003; Matos, \& Maler- 
bi, 2001), bem como a produção de conhecimento de cunho reflexivo ou conceitual (Matos, 1990, 1999a, 1999c, 2000).?

Assim, qualquer discussão ou mesmo a mera apresentação dos métodos de pesquisa em análise do comportamento deve abranger toda a diversidade de métodos que foram empregados nos 80 anos da disciplina. Esta diversidade de alternativas, no entanto, se dá dentro de parâmetros que trazem aos métodos de pesquisa na análise do comportamento especificidades metodológicas que os distinguem das práticas de outras disciplinas. Essa especificidade decorre das concepções de objeto de estudo, conhecimento, mundo e homem que informam a análise do comportamento. E esta especificidade deve muito, é claro, às características especiais da pesquisa experimental, de laboratório, na análise do comportamento. Esta é a modalidade de pesquisa que tem caráter fundador na análise do comportamento, representa o que pode ser considerado o paradigma metodológico da disciplina e marca todas as demais modalidades ou métodos de pesquisa quando estes são utilizados por analistas do comportamento. O paradigma é tão importante que um rótulo que o descreve - análise experimental - foi empregado como subtítulo do livro que represento a primeira sistematização da disciplina (The behavior of organisms) e foi, então, também tomado como o nome de toda a disciplina: análise experimental do comportamento. No entanto, ainda que seja comum encontrar em críticos da análise do comportamento, ou mesmo entre analistas do comportamento, uma redução da análise do comportamento à análise experimental do comportamento, e a consequente redução da pesquisa à pesquisa experimental, tal redução é, como vimos, inverídica.

Mas é verdadeiro que o compromisso com a pesquisa entendida de forma ampla e o forte compromisso com a experimentação estão entre as características mais positivas, a nosso ver, da análise do comportamento. A pesquisa básica na análise do comportamento, desde o trabalho inicial de Skinner, teve especificidades que produziram uma abordagem inédita à pesquisa experimental e um método experimental idiossincrático. Esta abordagem e suas especificidades marcaram todas as demais formas de conduzir pesquisa em análise do comportamento. Por esta razão, a seguir trataremos do que foi chamado de método do sujeito único em pesquisa experimental para depois introduzir algumas questões relacionadas a pesquisas não experimentais em análise do comportamento.

7 Há que destacar, ainda, no trabalho de Maria Amélia Matos, sua dedicação à institucionalização da análise do comportamento no país, através de seu envolvimento com o ensino, a formação de pesquisadores (Matos, 1996b, 1998) e a produção de material bibliográfico relevante para a formação em análise do comportamento (Danna \& Matos, 1999; Machado \& Matos, 1990; Matos \& Tomanari, 2002). 


\section{A pesquisa experimental nas ciências comportamentais}

A pesquisa experimental, obviamente, não é novidade em ciência ou nas chamadas ciências comportamentais ${ }^{8}$ e em subdisciplinas da biologia. O método experimental foi e continua sendo método preferencial em outras abordagens na psicologia e em outras ciências comportamentais, uma vez que como método permite predição e controle e descrições ou explicações mais potentes que qualquer outro método de pesquisa. A potência do método experimental fundamenta-se na noção de que fenômenos são determinados ou se relacionam com outros fenômenos de maneira regular. No trabalho de pesquisa define-se o fenômeno de interesse e dimensões (variáveis) características destes fenômenos são mensuradas. Então, manipula-se um segundo conjunto de fenômenos (variáveis) que, supostamente, se relacionam/determinam o conjunto de fenômenos mensurado e, mais uma vez, mensura-se (dimensões) o fenômeno de interesse. Se manipulações e medidas originam resultados consistentes, assume-se uma relação de dependência entre as variáveis manipuladas e mensuradas. Outros métodos de pesquisa não permitem a mesma replicabilidade e o mesmo controle de variáveis, portanto, não têm tem a mesma potência preditiva da pesquisa experimental.

Apesar da potência dos métodos experimentais, desde há muito constatou-se nas ciências comportamentais que os cânones e procedimentos das ciências físicas não poderiam ser simplesmente transferidos a problemas de pesquisa envolvendo questões relativas a comportamentos (Campbell \& Stanley, 1966; Cozby, 2003; Kerlinger, 1964; Reaves, 1992). Os problemas com a aplicação do método experimental às ciências comportamentais estão relacionados a um conjunto de suposições que, de uma maneira geral, fundamentam filosófica e epistemologicamente estas ciências: a suposição de multideterminação dos fenômenos de interesse, de sua historicidade (ou seja, a suposição de que os fenômenos relacionados a comportamentos constituem-se no tempo) e que pelo menos parte da variabilidade mensurada na experimentação é inerente e intrínseca ao fenômeno e, daí, não determinada e, portanto, não controlável. Estas características tornam difícil a pesquisa experimental, que é "aquela parte da pesquisa em que variáveis são manipuladas e os efeitos sobre outras variáveis são observados" (Campbell \& Stanley, 1966, p. 7) e as primeiras dificuldades são hoje reconhecidas também nas ciências exatas.

8 Os termos ciências comportamentais e ciências humanas serão utilizados aqui como intercambiáveis para referir as disciplinas que tomam como objeto de estudo, genericamente, algum aspecto relacionado direta ou indiretamente a comportamento. Entre as ciências chamadas comportamentais estariam, por exemplo, a sociologia, economia, antropologia, história, e a psicologia. Já os termos ciências físicas e exatas também serão utilizados como se fossem sinônimos e se referem às ciências mais antigas e nas quais os métodos experimentais tiveram origem, como a física (e suas subdisciplinas) ou a química. 
Mas a questão da variabilidade foi entendida de modo muito particular nas ciências comportamentais e nestas ciências duas "soluções" para o problema foram construídas. Uma solução encontrada por algumas disciplinas ou correntes e vertentes de disciplinas foi abandonar toda tentativa de pesquisa experimental, alegando que a pesquisa experimental destrói o fenômeno de interesse e assumindo, muitas vezes, que predição e controle não são relevantes em ciência. Por outro lado, porque se assumiu a variabilidade como intrínseca ao fenômeno estudado, foram desenvolvidos procedimentos para separar, ou distinguir, nos resultados, se e quanto as diferenças entre grupo controle e experimental representam variabilidade experimentalmente relevante e o quanto a variabilidade intrínseca dos fenômenos comportamentais está presente nos resultados. Estes procedimentos e técnicas, mais uma vez, foram inspirados pelas ciências exatas: buscou-se na estatística inferencial a solução para lidar com a questão da variabilidade obtida nos resultados da pesquisa experimental (Johnston, 1988; Johnston \& Pennypacker, 1993a, 1993b).

Assim, o método experimental, tal como foi consagrado nas ciências comportamentais, caracteriza-se como um conjunto de procedimentos nos quais os mais importantes aspectos metodológicos são:a amostragem e a participação de grandes números de sujeitos; sua distribuição em dois grupos, chamados de grupo experimental e grupo controle; a "atribuição randômica" dos sujeitos nos grupos; e o tratamento estatístico dos resultados. A alocação randômica dos participantes relaciona-se diretamente à possibilidade de tratamento estatístico dos resultados e é tão importante nesta perspectiva, que Campbell e Stanley (1966) consideraram como "experimentos verdadeiros" (true experiments) apenas aqueles delineamentos que prevêem tal distribuição (Campbell \& Stanley, 1966; Todman \& Dugard, 2001) e chamaram de quase-experimentos (quasi-experiments) todos os demais delineamentos de pesquisa experimental.

\section{A pesquisa experimental na análise do comportamento e os delineamentos de sujeito único}

Como salientou Matos (1990), nas ciências comportamentais o controle experimental foi substituído pelo controle estatístico, mesmo nos delineamentos de pesquisa experimental. Mas a análise do comportamento é uma exceção à regra e desenvolveu uma forma particular de experimentação promovida pela sua melhor prática de pesquisa, sustentada em supostos sobre o objeto de estudo de interesse que eram distintos daqueles mais comumente assumidos nas ciências comportamentais. al prática foi denominada de modelo, ou de delineamentos, do sujeito único ou de $n=1$ e, em 1990, Maria Amélia Matos apresentou uma síntese deste modelo que dificilmente poderá ser superada. ${ }^{9}$

9 Uma descrição detalhada das características específicas dos delineamentos de sujeito como 
Os supostos que conduziram à perspectiva única e peculiar que caracteriza a pesquisa experimental em análise do comportamento são: (1) comportamento é um fenômeno "natural", ou seja, submetido a leis, ou, ainda, passível de descrição, previsão e controle; (2) comportamento é um fenômeno que ocorre no indivíduo; (3) comportamento envolve classes de interações, ou seja, classes de respostas e de estímulos e não instâncias únicas; (4) comportamento é definido como interações indivíduo-ambiente, portanto os sujeitos individuais são sensíveis a alterações ambientais e se modificam nas suas interações com o ambiente; (5) as relações comportamentais são relações funcionais entre estímulos (ambiente) e respostas (atividade do organismo) e, portanto, as descrições topográficas são de interesse secundário; (6) comportamento é um fenômeno histórico e relacional; (7) a descrição/explicação do comportamento envolve a descrição de processos; (8) a pesquisa experimental é pesquisa de processo e são estes processos que emprestam regularidade ao fenômeno comportamental (Johnston \& Pennypacker, 1993a, 1993b; Matos, 1990; Sidman, 1960, Skinner, 1947; 1950). Finalmente, (9) enquanto em muitas disciplinas e correntes nas ciências comportamentais a variabilidade é classificada de duas maneiras, distinguindo-se variabilidade que é significativa para o problema de pesquisa e variabilidade que é típica do fenômeno, na pesquisa em análise do comportamento a variabilidade nos resultados experimentais foi sempre tomada como questão de controle experimental e não implicou na suposição de que a variabilidade obtida experimentalmente estaria além do alcance da investigação científica.

Os programas de pesquisa na análise do comportamento, bem como os seus métodos, estão baseados em tais suposições e se originam do trabalho de Skinner já nos anos 1930. As práticas de pesquisa experimental, de laboratório de Skinner e, no decorrer dos anos, de gerações de pesquisadores, como já se destacou, geraram um corpo explanatório sobre o comportamento que assumiu características de uma disciplina científica: a análise do comportamento. Geraram também, o que é de interesse especial aqui, procedimentos específicos até hoje empregados em laboratórios de análise do comportamento. Estes procedimentos, algumas vezes identificados como pesquisa operante, envolvem experimentação na qual se manipulam condições ambientais, mensurando seus efeitos sobre o responder de organismos individuais. As (classe de) respostas são experimentalmente definidas e selecionadas e podem ser dependentes ou não das alterações ambientais que seguem e/ou antecedem temporalmente o responder de um dado organismo.

\footnotetext{
seu próprio controle não cabe aqui e há na literatura de análise do comportamento excelentes fontes. Sidman (1960) apresentou magistralmente o "método" e desde então acumulase uma literatura razoável em relação ao tema dirigida ao pesquisador interessado. Alguns exemplos são: Bailey \& Burch, 2002; Hersen \& Barlow,1976; Johnston \& Pennypacker, 2003a, 2003b; Kazdin, 1982, 1992.
} 
Respeitando o caráter individual, interacional, processual e histórico do comportamento, Skinner, e outros que o sucederam na mesma tradição, optaram por experimentos com delineamentos de sujeito único. Nestes experimentos, como afirmou Sidman (1960), bastaria um sujeito porque em vez de uma ou poucas observações de muitos sujeitos, cada um deles em uma só condição (experimental ou controle), como é típico dos delineamentos experimentais de grupo, um sujeito é submetido às condições de interesse. E em vez de uma ou poucas medidas em uma condição são feitas repetidas medidas de seu comportamento nas sucessivas condições. Em geral os estudos experimentais em análise do comportamento, então, são delineados com poucos organismos, mas se caracterizam como longitudinais no âmbito do comportamento estudado: classes de respostas, escolhidas muitas vezes exatamente por seu caráter arbitrário, ${ }^{10}$ são selecionadas e mantidas por sucessivas condições experimentais. A apresentação das condições experimentais depende dos desempenhos obtidos e variabilidade e estabilidade nas medidas são fundamentais para a tomada de decisão na condução do experimento e na análise dos resultados. Assim, o responder de um organismo é estudado extensamente, no decorrer do tempo e de condições experimentais. Para tanto, são escolhidas (dimensões de) repostas e são manipuladas condições experimentais que permitem, em princípio, a recorrência de repostas, no tempo. Em vez de controlar a possibilidade de emissão de respostas em momentos dados e específicos do experimento, utilizando procedimentos do tipo "tudo ou nada" (procedimentos de uma tentativa em que o responder ocorre ou não), respeita-se o caráter processual e de fluxo do comportamento, com procedimentos que possibilitam a ocorrência e recorrência de repostas livremente (procedimentos de operante livre), medindo-se dimensões do responder que seriam afetadas no decorrer do processo de interação organismo-ambiente: a taxa de respostas, a medida mais paradigmática da pesquisa operante, tem estas características. $\mathrm{O}$ que importa aqui é que $\mathrm{O}$ sujeito funciona como controle dele mesmo e é isso que se quer destacar com a expressão delineamento de sujeito único.

Mais uma vez, como salientou também Matos(1990), os experimentos com delineamento de sujeito único envolvem também o compromisso com a análise de dados do comportamento individual. Mesmo que vários sujeitos tenham sido submetidos ao mesmo conjunto de condições, os resultados são tratados individualmente. No lugar de análises estatísticas sobre os desempenhos de diferentes sujeitos em distintas condições experimentais, são examinadas as medidas de desempenho de um sujeito no processo de interação com o ambiente experimental. A variabilidade é

10 Por exemplo, respostas como pressão à barra, no rato ou em macacos, que só são selecionadas e só podem ser emitidas na caixa experimental ocorrem fora da sessão experimental. $O$ seu caráter arbitrário significa ganho em termos do controle experimental, embora possa gerar preocupações com relação à generalidade. 
esperada quando condições experimentais (ambientais) são manipuladas e a estabilidade é tomada como produto de um processo de interação em um ambiente relativamente estável (Skinner, 1947, 1950, 1956). O foco da análise dos resultados na pesquisa experimental nos delineamentos de sujeito único se desloca para a análise das mudanças comportamentais produzidas no decorrer dos experimentos: em vez de sumarização estatística de dados que expressariam valores médios ou supostamente típicos em distintas condições, desenvolveram-se técnicas de análise de dados que permitem a avaliação das mudanças e da estabilização do responder, ou seja, uma avaliação de processo (Iversen, 1991). Por isso, em relatos de pesquisa de análise experimental do comportamento gráficos são mais comuns do que em outras tradições experimentais e, certamente, são mais importantes que testes estatísticos. Por isso também os gráficos envolvem, nos melhores casos, a representação do responder de um sujeito no decorrer do tempo e em função das manipulações experimentalmente realizadas. Os registros cumulativos criados por Skinner e largamente utilizados na análise experimental do comportamento são exemplares: permitem a visualização do fluxo comportamental em relação a uma classe de respostas. Nos registros cumulativos é possível "ver" o processo comportamental e não meramente o produto que resulta de um processo de interações. $A$ comparação de registros entre sujeitos ou de um mesmo sujeito, quando analisada tendo como parâmetro as operações ambientais, permite ver a regularidade de processo (taxas se aceleram ou desaceleram, mantêm-se estáveis ou não) sem escamotear as diferenças individuais - a taxa local e a taxa total pode ser diferente entre sujeitos ou em diferentes sessões (Morris \& Smith, 2004). Como salientou Matos (1990), os delineamentos de sujeito único não tomam os sujeitos "como grupos de sujeitos anônimos, anódinos, incolores e descontaminados" (p. 589).

Assim, desenvolveu-se na análise experimental do comportamento mais do que um conjunto de práticas específicas ou de técnicas de pesquisa, ${ }^{11}$ desenvolveu-se uma "filosofia" da experimentação em ciências comportamentais. No lugar de experimentos baseados em delineamento de grupos, delineamentos de sujeito único, do sujeito como seu próprio controle ou do $n=1$ caracterizam a pesquisa em análise experimental do comportamento; no lugar de atribuição randômica e estatisticamente determinada dos sujeitos em grupos, para distribuir igualmente entre os grupos a variabilidade indesejada, foram desenvolvidos delineamentos de pesquisas no quais o sujeito é seu próprio controle, ou seja, é submetido a todas ou, pelo menos, a mais de uma condição experimental e em que múltiplas medidas são tomadas; no lugar de tratamento estatístico de dados, tratamento visual e molecular dos dados.

11 Uma expressão em inglês para tal prática é lab lore. 


\section{Questões sobre a pesquisa experimental na análise do comportamento e os delineamentos de sujeito único}

A sistemática pesquisa experimental de processos, conduzida principalmente com delineamentos de sujeito único é marca da análise do comportamento, o que significa:os conceitos e a sistematização conceitual que conhecemos como análise do comportamento dependem diretamente dos métodos e resultados desta pesquisa e, por sua vez, provocaram, informaram e conduziram a experimentação. As relações entre teoria e práticas de pesquisa na análise do comportamento, como em qualquer ciência, são relevantes e merecem discussão porque desta discussão emergem novos programas de pesquisa ou avanços conceituais e também se originam questões e novas práticas metodológicas.

A história da análise do comportamento é também, então, a história de como se articulam e às vezes como se confrontam suposições que envolvem de um lado procedimentos metodológicos e de outro conceitos e pressupostos filosóficos. Não é possível listar ou discutir exaustivamente estas interações e confrontos que são dinâmicos, complexos e mutáveis. $O$ exercício de identificar, destacar e refletir "casos exemplares" desta interação deve contribuir para a identificação de dificuldades e de soluções para questões de método e de conceito na análise do comportamento.

Algumas das questões atuais relevantes em análise do comportamento se originam da própria história de pesquisa e teorização na análise do comportamento e têm implicações para a pesquisa atual e para o futuro da abordagem, por isso são brevemente apresentadas a seguir.

\subsection{A questão da reversibilidade comportamental.}

Os delineamentos de sujeito único em alguma medida supõem a possibilidade de reversibilidade do comportamento (experimentalmente mensurado) quando operações ambientais experimentalmente produzidas são retiradas. Esta suposição está implícita nos delineamentos de reversão e, mesmo, em outros, tais como os delineamentos de linha de base múltipla. No entanto, resultados experimentais como aqueles que envolvem as noções de contraste comportamental, learning set, ou ressurgência, por exemplo, demonstram que esta suposição é, pelo menos em certa medida, incorreta, como se esperaria da própria concepção de comportamento como processual e histórica (Bachá-Méndez, Reid, \& Mendoza-Soylovna, 2007; Fantino, Dunn, \& Mec, 1979; McSweeney \& Norman, 1979; Lieving \& Lattal, 2003; Milo, Odum, \& Shahan, 2003; Nevin, Reed, \& Morgan, 2006).

O que os resultados experimentais ensinam, neste caso, é que a reversibilidade comportamental não é um fenômeno a ser esperado necessariamente e não se pode confundir a reversibilidade que pode ser obtida experimentalmente e que diz respeito à emissão de instâncias de repostas com a reversibilidade de processo. A reversão de um comportamento ou 
classe de respostas em um experimento, constatada pelo retorno a níveis de medida semelhantes a aqueles obtidos antes de uma manipulação experimental diz respeito às condições ambientais momentâneas que são relevantes para a emissão de instâncias de respostas e não sobre a reversão dos processos selecionadores. O estudo de fenômenos como ressurgência ou contraste comportamental, portanto, representa um avanço em nossa compreensão do comportamento, uma vez que indica condições em que processos comportamentais vivenciados se manifestam, por exemplo (ver especialmente Johnston e Pennypacker, 1993b, e Sidman, 1960).

\subsection{A questão da variabilidade}

A proposição de que variabilidade no responder é ela mesma operante pode conduzir a uma discussão sobre se a variabilidade comportamental é intrínseca. No entanto, a "variabilidade operante" é um fenômeno da mesma natureza que outros fenômenos estudados na análise do comportamento.

Ou seja, quando a variabilidade é problema de pesquisa na análise do comportamento, ela mesma é conceituada como fenômeno sujeito a leis e afetado por alterações ambientais. Portanto, não é o mesmo que a suposta variabilidade intrínseca que seria característica dos seres vivos e que seria necessariamente não determinada. $O$ estudo da variabilidade comportamental, como processo operante, foi possibilitado exatamente pelos procedimentos e delineamentos de sujeito único e, assim, não representam uma ameaça metodológica (Sério, Andery \& Micheletto, 2005).

Além disso, o modelo de seleção por consequências (Skinner, 1981) supõe que seleção e variação estão envolvidos no fenômeno comportamental e as leis que descrevem ambos os processos são relevantes para nossa compreensão do comportamento. A pesquisa sobre variabilidade comportamental deve possibilitar o desenvolvimento de técnicas e procedimentos metodológicos, tais como qual a melhor maneira de identificar e mostrar regularidade na variabilidade e avanços conceituais aprofundando nossa compreensão do comportamento.

\subsection{A questão dos procedimentos de tentativas versus procedimentos de operante livre.}

Uma revisão dos periódicos dedicados a relatos de pesquisa experimental, básica ou aplicada, provavelmente mostrará um grande aumento na proporção de pesquisas que utilizam delineamentos com tentativas e não com operantes livres, especialmente na literatura sobre controle de estímulos (Hinson \& Higa, 1989). Consistentemente são medidas privilegiadas nestes estudos aquelas que envolvem ocorrência e não recorrência como PE o caso da taxa de respostas (ou frequência). Possivelmente estes delineamentos são mais coerentes com questões de pesquisa que envolvem a emissão de respostas e as funções evocativas, ou instanciadoras, do 
ambiente (Glenn \& Field, 1993, Michael, 1983, 1986). São estas as funções estudadas na pesquisa de controle de estímulos sobre o comportamento operante. $\mathrm{O}$ desafio que se apresenta para a pesquisa aqui é o de esclarecer que estas funções dependem da função selecionadora do ambiente (exercida pelo reforçamento).Também é um desafio tratar os resultados de procedimentos de tentativas, eles mesmos em termos de séries temporais, garantindo análise de dados que respeita o caráter processual das relações comportamentais (ver, por exemplo, Perone, M. (1991).

\subsection{Análise molecular versus análise molar}

Com a proposição de Herrnstein sobre esquemas concorrentes e escolha e com a Lei da Igualação proposta por Baum (Baum, 1979, 1981, 2002; Hale \& Shimp, 1975; Herrnstein, 1974; Herrnstein \& Loveland, 1976), surgiu uma distinção na análise experimental do comportamento entre o que se chamou de uma análise molar e uma análise molecular do comportamento. Se a distinção diz respeito à possibilidade de que a análise de resultados e mesmo os interesses de pesquisa sobre os fenômenos comportamentais podem recair sobre o efeito momentâneo e mais localizado de variáveis ambientais sobre comportamentos específicos ou pode focar os efeitos de mudanças comportamentais mais ampliadas, inclusive temporalmente, a distinção é produtiva e parece ser, ela mesma, resultado da postura mais analítica que foi dominante nas primeiras décadas de desenvolvimento da análise do comportamento. No desenvolvimento da análise do comportamento é de se esperar que descrições mais molares e que abarcam interações comportamentais mais complexas sejam possibilitadas e substituam - sem, no entanto, recusar como incompatíveis - descrições mais moleculares.

\subsection{A questão do tratamento estatístico e da quantificação dos resultados}

É cada vez mais frequente o tratamento estatístico de resultados nos relatos de pesquisa - básica e aplicada. $\mathrm{O}$ aumento se deve em parte à exigência de editores de periódicos que não são analistas do comportamento ou que procuram aumentar a exposição e o impacto da pesquisa em análise do comportamento junto à comunidade científica externa à disciplina. Também ganham espaço nas publicações de análise do comportamento tentativas de quantificação dos resultados, como maneira de expressar matematicamente regularidades comportamentais (Ator, 1999; Hopkins, Cole, \& Mason, 1998; Kollins, Newland, \& Critchfield, 1999; Shull, 1991).

Tratamentos estatísticos não são proibidos desde que não substituam a análise individual dos dados. Uma das características mais positivas e produtivas da pesquisa em análise do comportamento é que o pesquisador é controlado pelos resultados inclusive na condução da pesquisa, ou seja, que planos de pesquisa são alterados na medida em que os dados são 
produzidos. Esta prática não deve ser substituída, entretanto, por procedimentos padronizados e que afastam o experimentador do dado.

Por isso e porque o dado comportamental é um dado substantivo, o tratamento estatístico pode, com vantagens, complementar a análise individual, mas não pode ser seu substituto. Do mesmo modo, a quantificação de leis não é necessariamente um problema. $O$ problema existe quando e se a quantificação tornar o grande objetivo da pesquisa. A expressão matemática é exatamente isso: uma expressão. Ela deve refletor o dado, o fenômeno. Ela é produzida a partir do fenômeno e não o inverso (ver, por exemplo, Iversen \& Lattal, 1991b).

\title{
7.6 A questão do ser humano como participante de pesquisa.
}

Os dados de pesquisa que originaram o cerne conceitual da análise do comportamento foram produzidos em laboratório com sujeitos infrahumanos. Assumia-se então que os processos investigados não eram específicos de espécie: reforçamento, por exemplo, é o processo envolvido na seleção de comportamento operante nas diferentes espécies sensíveis a condicionamento operante. A pesquisa com participantes humanos, especialmente a partir dos anos 1960, gerou discussões sobre a suposição e, mais especialmente, sobre o papel que teria comportamento verbal como mediador necessário de todo comportamento humano. Se assumida, a tese invalidaria, em certa medida, o cerne dos resultados e dos modelos de pesquisa e das proposições teóricas da análise do comportamento. No entanto, a saudável prática de responder a perguntas com pesquisa parece ter produzido novos programas de investigação (por exemplo, comportamento verbal e controle verbal do comportamento, comportamentos emergentes e equivalência de estímulos) e os resultados destes programas vêm alargando nossa compreensão do comportamento nas mesmas bases (ver a este respeito, por exemplo, Lattal \& Perone, 1996).

A pesquisa com participantes humanos, baseada em delineamos de sujeito único, é um desafio a ser enfrentado nas áreas de comportamento social e comportamento verbal. Contingências entrelaçadas ainda envolvem contingências de reforçamento e a pesquisa nestas áreas precisa definir e distinguir as unidades analíticas apropriadas (Lattal \& Perone, 1998).

\section{Research methods in behavior analysis}

\begin{abstract}
The following topics are discussed: the status of behavior analysis as a scientific discipline and the related consequences to the characterization of research methods in behavior analysis. The array of research methods is classified according to distinct criteria, with an emphasis on experimental research. Underlying principles and main features of single case designs are presented.
\end{abstract}


Finally, some of the current conceptual-methodological issues in behavior analysis are briefly discussed, such as: behavioral reversibility, behavioral variability, trial and free operant procedures, molecular and molar analysis, statistics and quantification applied to single case procedures and experimental research with human participants.

Keywords: Behavior analysis. Method. Experimental research. Single-case designs.

\section{Méthodes de recherche en analyse du comportement}

Resumé: Cet article présente une discussion sur le status de l'analyse du comportement en tant que discipline scientifique, ainsi que le rapport de celui-la avec les autres sciences et ses implications pour la caractérisation des méthodes de recherche en analyse du comportement. Plusieurs classifications des méthodes de recherche sont présentés, mettant l'accent sur la méthode expérimentale, compte tenu de son rôle dans la construction de la discipline. Les présuppositions et les principales caractéristiques de dessin de sujet unique sont présentées. Enfin, sont en peu de mots discutés des questions qui découlent de l'interaction et de la confrontation apparente entre le concept et la pratique de la recherche dans l'analyse du comportement: réversibilité et variabilité comportementale, les procédures d'essai et opérant libre, l'analyse moléculaire et molaire, l'analyse statistique et la quantification, la recherche expérimentale avec des humains.

Mots clés: Analyse du Comportement. Méthodes. Recherche expérimentale. Dessin de sujet unique.

\section{Métodos de investigación en Análisis del Comportamiento}

Resumen: Este artículo presenta una discusión sobre el estatus del Análisis del Comportamiento como disciplina científica, así como su relación con otras ciencias y las implicaciones de esta discusión para la caracterización de los métodos de investigación en Análisis del Comportamiento. Se presentan varias clasificaciones de los métodos de investigación, con énfasis en el método experimental, dado su papel en la construcción de la disciplina. Son presentados los supuestos y las principales características de los diseños de caso único. Finalmente, se discuten brevemente algunas cuestiones que surgen de la interacción y aparente confrontación entre la conceptualización y la práctica investigativa en Análisis del Comportamiento, tales como: reversibilidad del comportamiento, variabilidad del comportamiento, procedimientos de ensayo discreto y de operante libre, análisis molecular y molar, tratamiento estadístico y cuantificación e investigación experimental con humanos.

Palabras clave: Análisis del Comportamiento. Métodos. Investigación experimental. Diseño de caso único. 


\section{Referências}

Andery, M. A., \& Sério, T. M. (1987). Uma análise histórica do pensamento de B.F.Skinner. Anais da XVII Reunião Anual de Psicologia - SPRP, Ribeirão Preto.

Andery, M. A. P. A., \& Sério, T. M. A. P. (1989). O controle pela conseqüência no desenvolvimento da cultura. Psicologia. Teoria e Pesquisa,5, 149-155.

Andery, M. A. P. A. (1997). O modelo de seleção por conseqüências e a subjetividade. In R. Banaco (Org.), Sobre comportamento e cognição (Vol. 1). Santo André, SP: ARBytes.

Andery, M. A. P. A., Micheletto, N., \& Sério, T. M. A. P. (2002). Uma análise das referências feitas por Skinner de 1930 a 1938. Revista Brasileira de Terapia Comportamental e Cognitiva, 4 (1), 21-33.

Ator, N. A. (1999). Statistical inference in behavior analysis: Environmental determinants? The Behavior Analyst, 22, 93-97.

Bachá-Méndez, G., Reid, A. K., \& Mendoza-Soylovna, A. (2007). Resurgence of integrated behavioral units. Journal of the Experimental Analysis of Behavior, 87, $5-24$.

Bailey, J. S., \& Burch, M. B. (2002). Research methods in applied behavior analysis. Thousand Oaks, CA: Sage.

Baum, W. M. (1979). Matching, undermatching, and overmatching in studies of choice. Journal of the Experimental Analysis of Behavior, 32, 269-281.

Baum, W. M. (1981). Optimization and the matching law as accounts of instrumental behavior. Journal of the Experimental Analysis of Behavior, 36, 387-403.

Baum, W. M. (2002). From molecular to molar: A paradigm shift in behavior analysis. Journal of the Experimental Analysis of Behavior, 78, 95-116.

Blackman, D. E. (1991). B.F. Skinner and G. H. Mead: On biological science and social science. Journal of the Experimental Analysis of Behavior, 55, 251-265.

Campbell, D. T., \& Stanley, J. C. (1966). Experimental and quasi-experimental designs for research. Boston, MA: Houghton Mifflin.

Catania, A. C. (1973). The concept of the operant in the analysis of behavior. Behaviorism, 1, 103-115. 
Catania, A. C. (1984a). Problems of selection and phylogeny, terms and methods of behaviorism. Behavioral and Brain Sciences, 7, 713-717.

Catania, A. C. (1984b). The operant behaviorism of B. F. Skinner. Behavioral and Brain Sciences, 7, 473-475.

Catania, A. C. (1999). Learning. New York: Sloane.

Coleman, S. R. (1985). B. F. Skinner, 1926-1928: From literature to psychology. The Behavior Analyst, 8(1), 77-92.

Coleman, S. R. (1987). Quantitative order in B. F. Skinner's. The Behavior Analyst, 10, 47-65.

Cozby, P. C. (2003). Métodos de pesquisa em ciências do comportamento. São Paulo: Atlas.

Danna, M. F., \& Matos, M. A. (1999). Ensinando observação: uma introdução (6a ed.). São Paulo: Edicon.

Davison, M., McCarthy, D., \& Jensen, C. (1985). Component probability and component reinforcer rate as biasers of free-operant detection. Journal of the Experimental Analysis of Behavior, 44, 103-120.

de Rose, J. C. (1993). Classes de estímulos: Implicações para uma análise comportamental da cognição. Psicologia: Teoria e Pesquisa, 9, 2, 283-303.

Debert, P., Matos, M.A., \& Mcllvane, W. (2007). Conditional relations with compound abstract stimuli using a go/no-go procedure. Journal of the Experimental Analysis of Behavior, 87, 89-96.

Fantino, E., Dunn, R., \& Meck, W. (1979). Percentage reinforcement and choice. Journal of the Experimental Analysis of Behavior, 32, 335-340.

Fraley, L. E., \& Vargas, E. A. (1986). Separate disciplines: The study of behavior and the study of the psyche. The Behavior Analyst, 9, 47-59.

Glenn, S. S. (2003). Operant contingencies and the origins of culture. In K. A. Lattal \& P. N. Chase (Eds.), Behavior theory and philosophy. New York: Kluwer Academic.

Glenn, S.S., \& Field, D. P. (1994). Functions of the environment in behavioral evolution. The Behavior Analyst, 17, 241-259. 
Hale, J. M., \& Shimp, C. P. (1975). Molecular contingencies and reinforcement probability. Journal of the Experimental Analysis of Behavior, 24, 315-321.

Herrnstein, R. J., \& Loveland, D. H. (1976). Matching in a network. Journal of the Experimental Analysis of Behavior, 26, 143-153.

Herrnstein, R. J. (1974). Formal properties of the matching law. Journal of the Experimental Analysis of Behavior, 21, 159-164.

Hersen, M., \& Barlow, D. H. (1976). Single case experimental designs. New York: Pergamon.

Hinson, J. M., \& Higa, J. J. (1989). Discrete and continuous measures of dimensional stimulus control. Journal of the Experimental Analysis of Behavior, 51, 199-214.

Hopkins, B. L., Cole, B. L., \& Mason, T. L. (1998). A critique of the usefulness of inferential statistics in behavior analysis. The Behavior Analyst, 21, 125-137.

Hull, D. L., Langman, R. E., \& Glenn, S. S. (2001). A general account of selection: Biology, immunology and behavior. Behavioral and Brain Sciences, 25, 511-528

Iversen, I. H. (1991). Methods of analyzing behavior patterns. In I. H. Iversen \& K. A. Lattal (Eds.), Experimental analysis of behavior. Part 2 (pp. 193-242) New York: Elsevier.

Iversen, I. H., \& Lattal, K. A. (1991a). Experimental analysis of behavior: Vol.6, Part 1. Amsterdam: Elsevier.

Iversen, I. H., \& Lattal, K. A. (1991b). Experimental analysis of behavior: Vol.6, Part 2. Amsterdam: Elsevier.

Johnston, J. M. (1988). Strategic and tactical limits of comparison studies. The Behavior Analyst, 11, 1-9.

Johnston, J. M., \& Pennypacker, H. S. (1993a). Strategies and tactics of behavioral research (2a Ed.). Hillsdale, NJ: Lawrence Erlbaum.

Johnston, J. M., \& Pennypacker, H. S. (1993b). Readings for Strategies and tactics of behavioral research (2a Ed.). Hillsdale, NJ: Lawrence Erlbaum.

Kazdin, A. E. (1982). Single-case research designs. New York: Oxford University Press.

Kazdin, A.E. (1992). Methodological issues and strategies in clinical research. Washington, DC: American Psychological Association. 
Keller, F. S., \& Schoeenfeld, N. (1950). Principles of psychology. New York: Appleton Century Crofts.

Kerlinger, F. N. (1964). Foundations of behavioral research. New York, NY: Holt, Rinehart \& Winston.

Kollins, S. H., Newland, M. C., \& Critchfield, T. S. (1999). Quantitative integration of single-subject studies: Methods and misinterpretations. The Behavior Analyst, 22, 149-157.

Lattal, K. A., \& Perone, M. (1998). Handbook of research methods in human operant behavior. New York: Plenum Press.

Lieving, G.A., \& Lattal, K. A. (2003). Recency, repeatability, and reinforcer retrenchment: An experimental analysis of resurgence. Journal of the Experimental Analysis of Behavior, 80, 217-233.

Luna, S. V. (2007). Planejamento de pesquisa. São Paulo: EDUC.

Machado, L. M. C. M., \& Matos, M. A.(1990). O laboratório em cursos de graduação em psicologia: buscando mudar atitudes. Ciência e Cultura, 42, 647652.

Matos, M. A. (1969). Acquisition and extinction of conditioned suppression in the rhesus monkey as a function of probability of unavoidable shock. Tese de Doutorado, Columbia University, New York.

Matos, M. A. (1981a). A ética do exercício de controles aversivos. Boletim de Psicologia, $33,125-133$.

Matos, M. A. (1981b). O controle de estímulos sobre o comportamento. Psicologia, 7, $1-15$.

Matos, M. A. (1990). Controle experimental e controle estatístico: a filosofia do caso único na pesquisa comportamental. Ciência e Cultura, 42, 585-592.

Matos, M. A. (1996). Contingências para a análise comportamental no Brasil. Psicologia: Teoria e Pesquisa, 12, 107-111.

Matos, M. A. (1998). Carolina Martuscelli Bori: uma cientista brasileira. Psicologia: Reflexão e Crítica, 11, 411-420.

Matos, M. A. (1999a). Com o que o behaviorismo radical trabalha? In R. A. Banaco (Ed.), Sobre comportamento e cognição (pp. 45-53). Santo André, SP: ARBytes.

PsIcologia USP, São Paulo, abril/junho, 2010, 21(2), 313-342. 
Matos, M. A. (1999b). Controle de estímulo condicional, formação de classes conceituais e cognição. Revista Brasileira de Terapia Comportamental e Cognitiva, $1,158-178$.

Matos, M. A. (1999c). O behaviorismo metodológico e suas relações com o mentalismo e o behaviorismo radical. In R. A. Banaco (Ed.), Sobre comportamento e cognição (pp. 54-67). Santo André, SP: ARBytes.

Matos, M. A. (1999d). Leitura generalizada: Procedimentos e resultados? Em R. A. Banaco (Ed.), Sobre comportamento e cognição (pp. 470- 487). Santo André, SP: ARBytes.

Matos, M. A. (2000). Análise funcional do comportamento. Estudos de Psicologia, 16, 8-18.

Matos, M. A., \& Hubner, M. M. (1992). Equivalence relations and reading. In S. C. Hayes \& L. J. Hayes (Eds.), Understanding verbal relations (pp. 83-94). Reno, NV: Context.

Matos, M. A, Hubner, M. M. C., Peres, W., \& Malheiros, R. H. S., (1997). Oralização e cópia: efeitos sobre a aquisição de leitura generalizada recombinativa. Temas em Psicologia, 1, 47-65.

Matos, M. A., Hubner, M. M., Serra, V. R. B. P., Basaglia, A. E., \& Avanzi, A. L. (2003). Redes de relações condicionais e leitura recombinativa: pesquisando o ensinar a ler. Arquivos Brasileiros de Psicologia, 54, 284-303.

Matos, M. A., \& Lopes Júnior, J. (1999). Controle contextual e equivalência de estímulos Acta Comportamentalia, 7, 117-146.

Matos, M. A., \& Malerbi, F. E. K. (2001). Blood glucose discrimination training: The role of internal and external cues. Journal of Health Psychology, 6, 243-253.

Matos, M. A., Paracampo, C., Souza, D. G., \& Albuquerque, L. C. (2001). Efeitos do comportamento verbal e não verbal sobre as mudanças de reforçamento. Acta Comportamentalia, 9, 31-55.

Matos, M. A., \& Tomanari, G. Y. (2002). A análise do comportamento no laboratório didático. São Paulo: Manole.

McSweeney, F. K., \& Norman, W. D. (1979). Defining behavioral contrast for multiple schedules. Journal of the Experimental Analysis of Behavior, 32, 457-461.

Michael, J. (1980). Flight from behavior analysis. The Behavior Analyst, 3, 1-22. 
Michael, J. (1983). Evocative and repertoire-altering effects of an environmental event. The Analysis of Verbal Behavior, 2, 19-21.

Michael, J. (1986). Repertoire-altering effects of remote contingencies. The Analysis of Verbal Behavior, 4, 10-18.

Micheletto, N., \& Sério, T. M. A. P. (2002). Temos behavior para tantos 'ismos'? In H. J. Guilhardi (Org.), Sobre comportamento e cognição (Vol. 9). Santo André,SP: ESETec.

Micheletto, N. (2000). Bases filosóficas da noção de relação funcional. Revista Brasileira de Terapia Comportamental e Cognitiva, 2, 115-121.

Micheletto, N., Andery, M. A. P. A., \& Sério, T. M. A. P. (2000). Pesquisa histórica em análise do comportamento. Temas de Psicologia, 8, 137 - 142.

Moore, J., \& Cooper, J. O. (2003). Some proposed relations among the domains of behavior analysis. The Behavior Analyst, 26, 69-84.

Moore, J. (2008). Conceptual foundations of radical behaviorism. Cornwall-on-Hudson, New York: Sloan.

Morris, E. K., \& Smith, N. G. (2004). On the origin and preservation of cumulative record in its struggle for life as a favored term. Journal of the Experimental Analysis of Behavior, 82, 357-373.

Morris, E. K., Todd, J. T., Midgley, B. D., Schneider, S. M., \& Johnson, L. M. (1990). The history of behavior analysis: Some historiography and a bibliography. The Behavior Analyst, 13, 131-158.

Moxley, R. A. (1989). Some historical relationships between science and technology within behavior analysis. The Behavior Analyst, 12, 45-57

Moxley, R. A. (2001). Sources for Skinner's pragmatic selectionism in 1945. The Behavior Analyst, 24, 201-212.

Moxley, R. A. (2002). Some more similarities between Peirce and Skinner. The Behavior Analyst, 25, 201-214.

Moxley, R. A. (2003). Some early similarities and later differences between Bertrand Russell and B. F. Skinner. The Behavior Analyst, 26, 111-130.

Moxley, R. A. (2005). Ernst Mach and B. F. Skinner: Their similarities with two traditions for verbal behavior. The Behavior Analyst, 28, 29-48.

Psıcologia USP, São Paulo, abril/junho, 2010, 21(2), 313-342. 
Moxley, R. A. (2007). Ultimate realities: Deterministic and evolutionary. The Behavior Analyst, 30, 59-77.

Neuringer, A. (1984). Melioration and self-experimentation. Journal of the Experimental Analysis of Behavior, 42, 397-406.

Neuringer, A. (2002). Operant variability: Evidence, functions, and theory. Psychonomic Bulletin Review, 9, 672-705.

Neuringer, A. (2004). Reinforced variability in animals and people: Implications for adaptive action. American Psychologist, 59, 891-906.

Nevin, J. A. Milo, J. Odum, A. L., \& Shahan, T. A. (2003). Accuracy of discrimination, rate of responding, and resistance to change. Journal of the Experimental Analysis of Behavior, 79, 307-321.

Perone, M. (1991). Experimental design in the analysis of free operant behavior. In I. H. Iversen \& K. A. Lattal (Eds.), Experimental analysis of behavior (Part 1, pp. 135172). New York: Elsevier.

Reaves, C. (1992). Quantitative research for the behavioral sciences. New York: John Wiley.

Reed, P., \& Morgan, T. A. (2006). Resurgence of response sequences during extinction in rats shows a primacy effect. Journal of the Experimental Analysis of Behavior, 86, 307-315

Schick, K. (1971). Operants. Journal of the Experimental Analysis of Behavior, 15, 413-423.

Sério, T. M. A. P., Andery, M. A. P. A., \& Micheletto, N. (2005). A noção de variabilidade na obra de B. F. Skinner. Acta Comportamentalia, 13,98-110.

Shull, R. L. (1991). Mathematical description of operant behavior: An introduction. In I. H. Iversen \& K. A. Lattal (Eds.), Experimental analysis of behavior (Part 2, pp. 243282). New York: Elsevier.

Sidman, M. (1960). Tactics of scientific research. New York: Basic Books.

Sidman, M. (1989). Coercion and its fallouts. Boston: Authors Cooperative.

Sidman, M. (1990). Equivalence relations: Where do they come from? In D. E. Blackman \& H. Lejeune (Eds.), Behaviour analysis in theory and practice: Contributions and controversies. Hillsdale: Erlbaum. 
Sidman, M. (1997). Equivalence: A theoretical or a descriptive model? Mexican Journal of Behavior Analysis, 23, 125-145.

Skinner, B. F. (1931). The concept of the reflex in the description of behavior. Journal of General Psychology, 5, 427-458.

Skinner, B. F. (1935). The generic nature of the concepts of stimulus and response. Journal of General Psychology, 12, 40-65.

Skinner, B. F. (1936). The verbal summator and a method for the study of latent speech. The Journal of Psychology, 2, 71-107.

Skinner, B. F. (1937). The distribution of associated words. Psychological Record, 1, 71-76.

Skinner, B. F. (1938a). The behavior of organisms: An experimental analysis. New York: Appleton-Century-Crofts.

Skinner, B. F. (1938b). The frequencies of occurrence of associated words. Psychological Bulletin, 35, 675.

Skinner, B. F. (1939). The alliteration in Shakespeare's sonnets: A study in literary behavior. Psychological Record, 3, 186-192.

Skinner, B. F. (1947). Experimental psychology. In W. Dennis (Ed.), Current trends in psychology. Pittsburgh, PA: University of Pittsburgh Press.

Skinner, B. F. (1953). Science and human behavior. New York: The Free Press.

Skinner, B. F. (1956). A case history in scientific method. The American Psychologist, $11,221-233$.

Skinner, B. F. (1957). Verbal behavior. New York: Appleton-Century-Crofts.

Skinner, B. F. (1959). Teaching machines. Science, 128, 969-977.

Skinner, B. F. (1961). Teaching machines. Scientific American, 205, 90-102.

Skinner, B. F. (1968). The technology of teaching. New York: Appleton- Century-Crofts.

Skinner, B. F. (1972). The control of human behavior. In B. F. Skinner, Cumulative record. Acton, MA: Copley. (Trabalho original publicado em 1955)

Skinner, B. F. (1974). About behaviorism. New York: Alfred A. Knopf. 
Skinner, B. F. (1983). Intellectual self-management in old age. American Psychologist, $38,239-244$.

Skinner, B. F. (1981). Selection by consequences. Science, 213, 501-504.

Smith, T. L. (1994). Behavior and its causes: Philosophical foundations of operant psychology. Boston, MA: Kluwer.

Todd, J. T., \& Morris, E. K. (1995). Modern perspectives on B. F. Skinner and contemporary behaviorism. Reno, NV: Greenwood Press.

Todman, J. B., \& Dugard, P. (2001). Single-case and small-n experimental designs. New York: Lawrence Erlbaum.

Tourinho, E. Z. (1999). Estudos conceituais na análise do comportamento. Temas em Psicologia, 7(3), 213-222.

Tourinho, E. Z. (2003). A produção de conhecimento em psicologia: a análise do comportamento. Psicologia: Ciência e Profissão, 23(2), 30-41.

Tourinho, E. Z. (2006). Relações comportamentais como objeto da psicologia: algumas implicações. Interação, 1(1), 1-8.

Tourinho, E. Z., Carvalho Neto, M. B., \& Neno, S. (2004). A psicologia como campo de conhecimento e como profissão de ajuda. Estudos de Psicologia, 9(1), 17-24.

Vargas, E. A. (1987). "Separate disciplines" is another name for survival. The Behavior Analyst, 10, 119-121.

Vargas, J. S. (1989). The road less traveled by. The Behavior Analyst, 12, 121-130.

Zentall, T. R., \& Smeets, P. M. (1996). (Eds.), Stimulus class formation in humans and animals. Amsterdam: Elsevier.

Maria Amalia Pie Abib Andery, Pontifícia Universidade Católica de São Paulo. Rua Monte Alegre, 1352. CEP: 15014-002. São Paulo-SP. Endereço eletrônico: mandery@ pucsp.brou mandery@uol.com.br

Recebido em: 16/03/2010

Aceito em: 14/05/2010 